\title{
Predicting the Postoperative Outcome of Percutaneous Nephrolithotomy with Machine Learning System: Software Validation and Comparative Analysis with Guy's Stone Score and the CROES Nomogram
}

\author{
Alireza Aminsharifi, MD,,2 Dariush Irani, MD, Sona Tayebi, MD,, Taher Jafari Kafash, MD,1 \\ Tayebeh Shabanian, MS, and Hossein Parsaei, $\mathrm{PhD}^{3,4}$
}

\begin{abstract}
Purpose: To validate the output of a machine learning-based software as an intelligible interface for predicting multiple outcomes after percutaneous nephrolithotomy (PCNL). We compared the performance of this system with Guy's stone score (GSS) and the Clinical Research Office of Endourological Society (CROES) nomogram. Patients and Methods: Data from 146 adult patients (87 males, 59\%) who underwent PCNL at our institute were used. To validate the system, accuracy of the software for predicting each postoperative outcome was compared with the actual outcome. Similarly, preoperative data were analyzed with GSS and CROES nomograms to determine stone-free status as predicted by these nomograms. A receiver operating characteristic (ROC) curve was generated for each scoring system, and the area under the ROC curve (AUC) was calculated and used to assess the predictive performance of all three models.

Results: Overall stone-free rate was 72.6\% (106/146). Forty of 146 patients (27.4\%) were scheduled for 42 ancillary procedures (extracorporeal shockwave lithotripsy [SWL] $[n=31]$ or repeat PCNL $[n=11]$ ) to manage residual renal stones. Overall, the machine learning system predicted the PCNL outcomes with an accuracy ranging between $80 \%$ and $95.1 \%$. For predicting the stone-free status, the AUC for the software $(0.915)$ was significantly larger than the AUC for GSS $(0.615)$ or CROES nomograms $(0.621)(p<0.001)$.

Conclusion: At the internal institutional level, the machine learning-based software was a promising tool for recording, processing, and predicting outcomes after PCNL. Validation of this system against an external dataset is highly recommended before its widespread application.
\end{abstract}

Keywords: percutaneous nephrolithotomy, artificial intelligence, machine learning, support vector machines, renal calculus

\section{Introduction}

$\mathrm{N}$ THE ERA of minimally invasive surgery, percutaneous nephrolithotomy (PCNL) remains the standard of care for managing large renal calculi. ${ }^{1,2}$ Along with the widespread use of PCNL, several groups have recently proposed and tested scoring systems to predict stone-free status after PCNL ${ }^{3-5}$ Predictive models are potentially useful to facilitate clinical decision making and patient counseling. To predict the stone-free rate (SFR) after PCNL, Thomas and colleagues were the first to introduce Guy's stone score (GSS), a simple qualitative grading scale (grade I-IV) based on stone shape and configuration, as well as the presence or absence of renal or skeletal anomalies. ${ }^{3}$ This approach further evolved when Smith and colleagues designed a quantitative scoring nomogram: the Clinical Research Office of the Endourological Society (CROES) nomogram. ${ }^{4}$ To predict SFR after PCNL, several stone characteristics (burden, number, location, multiple, staghorn) together with institute-level case volume are included in this nomogram. A notable

\footnotetext{
${ }^{1}$ Department of Urology, Shiraz University of Medical Sciences, Shiraz, Iran.

${ }^{2}$ Laparoscopy Research Center, Shiraz University of Medical Sciences, Shiraz, Iran.

${ }^{3}$ Department of Medical Physics and Engineering, Shiraz University of Medical Sciences, Shiraz, Iran.

${ }^{4}$ Shiraz Neuroscience Research Center, Shiraz University of Medical Sciences, Shiraz, Iran.
} 
feature of the CROES method is that it considers the weighting of each parameter.

Recently, our group designed a machine learning-based system to predict post-PCNL outcomes such as SFR and the need for ancillary procedures. ${ }^{6}$ More than 20 preoperative and intraoperative variables and their relative weights are involved in this intelligent network. Preliminary work showed that this system showed promising accuracy $(81.0 \%-$ $98.2 \%$ ) in predicting stone-free status and the need for blood transfusion or ancillary procedures. ${ }^{6}$ Based on machine learning algorithms, we then designed software and a userfriendly interface (Fig. 1).

In this study, we sought to validate the output of this machine learning-based toolkit as an intelligible interface able to predict post-PCNL outcomes. We then compared the performance and accuracy of this system with GSS grading and CROES nomograms as two widely used prognostic tools for post-PCNL stone-free status. To our knowledge, this is the first comparative analysis of an artificial intelligence system designed to contrast its performance in predicting PCNL outcomes with currently available approaches.

\section{Patients and Methods}

\section{Ethics}

Approval for this study was obtained from our institutional review board. All patients were informed about the aims of this study and provided their informed consent to take part. All information imported into the software was de-identified and coded. Two-factor authentication was used for data encryption. Only authorized users had access to the information recorded in our system.

\section{Patients}

Data for all adult patients who underwent PCNL between September 2016 and November 2017 at our institute were included. All procedures were done by two fully qualified, competent endourologists (A.A., D.I.). Patients with bilateral PCNL or chronic kidney disease were excluded. All patients had CT scan as a part of preoperative imaging studies. Before all procedures, normal preoperative coagulation profile and negative urine culture were verified, and a single dose of intravenous ceftriaxone was administered at the time of anesthesia. All procedures consisted of standard fluoroscopyguided PCNL with the patient in the prone position. ${ }^{7} \mathrm{~A}$ pneumatic lithotripter was used for stone fragmentation, and a $14 \mathrm{~F}$ nephrostomy was placed in the collecting system at the end of surgery.

Postoperative stone-free status was assessed by CT scan, and it was defined as the absence of residual stones larger than $4 \mathrm{~mm}^{8}$ Major surgical complications and the need for ancillary procedures to manage residual stones were also recorded. The preoperative, intraoperative, and postoperative variables used in this study are summarized in Supplementary Table S1.

\section{Design and validation of machine learning-based software}

The machine learning technique used for data analysis, classification, and regression as well as for identifying the connections between input and output variables is based on the support vector machine (SVM) model. ${ }^{6,9,10}$ The SVM networks have an efficient training phase and are accurate, especially for clean datasets with well-defined input and

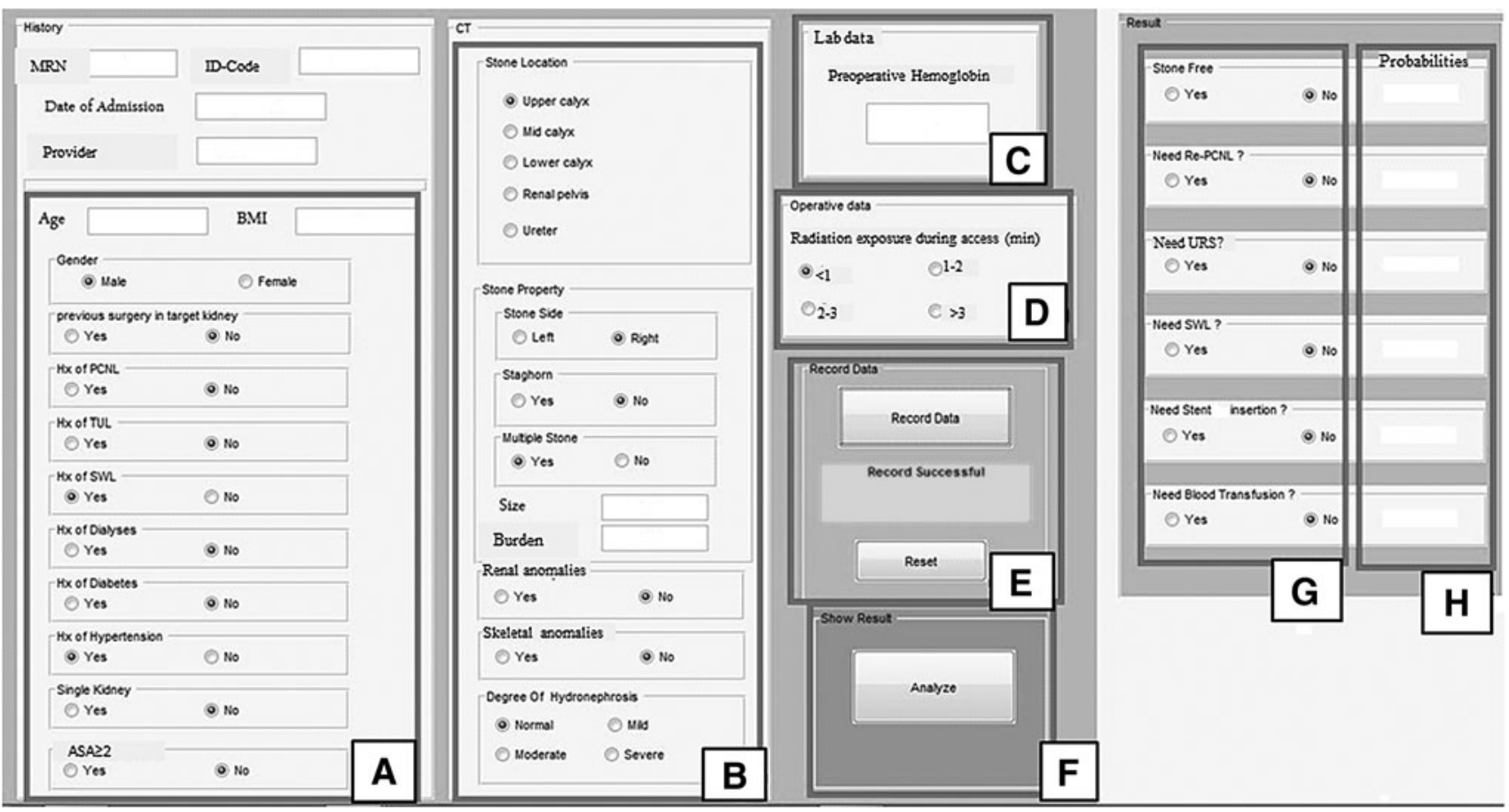

FIG. 1. The digital interface of the machine learning-based software consists of different panels for importing preoperative and intraoperative data $(\mathbf{A}-\mathbf{D})$, panels for recording and computing data $(\mathbf{E}, \mathbf{F})$, and the output panel for predicting multiple postoperative outcomes $(\mathbf{G}, \mathbf{H})$. 
output variables. ${ }^{9,10}$ Figure $2 \mathrm{~A}-\mathrm{C}$ illustrate this model when two or more variables are to be classified and analyzed. The software is rendered in MATLAB (Mathworks, Natick, MA) and its user interface is shown in Figure 1. The user can easily import the preoperative values into the appropriate panels, and after computation, the predicted outcomes can be extracted from the output panel. The software can also be used as a registry to record and retrieve data in different formats. Technical details of the development of machine learning tools used in the software were described earlier. ${ }^{11}$

\section{Clinical application and validation of the software}

Preoperative data for 146 adult patients were consecutively imported into the software, and its output was extracted. To validate the system, the accuracy of the software for predicting each postoperative outcome was compared with the actual outcome.

Similarly, preoperative data were analyzed with GSS grades and CROES nomogram, and stone-free status was predicted by these nomograms. ${ }^{3,4}$ The predictive performance of the nomograms was then calculated. A receiver operating characteristic (ROC) curve was generated for each scoring system, and the area under the ROC curve (AUC) was calculated and used to assess the predictive accuracy of nomograms versus the machine learning software. As expected, the GSS and CROES nomograms can directly predict post- operative stone-free status, but they are unable to directly capture other post-PCNL outcomes.

Participating surgeons were blinded to data collection and input into the nomograms and software. Each predictive model was used in its own Excel table, and separate teams processed each model.

\section{Results}

During the study period, 146 patients (87 males, 59\%) were enrolled. Mean age was $49.3 \pm 12.6$ years, and mean stone burden was $451.2 \pm 427.8 \mathrm{~mm}^{2}$. The demographic, preoperative, and intraoperative data for this cohort are summarized in Table 1. Mean hospital stay was $2.87 \pm 0.69$ days, during which the presence of postoperative residual stones was determined with noncontrast CT scans. All patients were discharged after removal of their nephrostomy tube. Table 2 shows the actual postoperative data for these patients. Overall SFR was $72.6 \%$ (106/146). Prolonged urine leakage requiring ureteroscopy (URS) and Double-J stent placement occurred in 12 patients (8.2\%). Postoperative blood transfusion due to significant blood loss was required in $11(7.5 \%)$. Forty of 146 patients $(27.4 \%)$ were scheduled for 42 ancillary procedures (extracorporeal shockwave lithotripsy [SWL] $(n=31)$ or repeat PCNL $[n=11])$ to manage residual renal stones (Table 2 ).

After preoperative data were analyzed and PCNL outcomes were predicted by the software, we then compared the
FIG. 2. As a supervised machine learning algorithm, SVM is based on finding hyperplanes to classify datasets (A). The SVM constructs a hyperplane or a set of hyperplanes in a high- or indefinite-dimension space that can be used for tasks such as classification, regression, or outlier detection. Support vectors represent the data points nearest to the hyperplane. These vectors are the critical elements of the dataset, because their elimination would alter the position of the dividing plane. Intuitively, the further away from the hyperplane the data points are, the greater the confidence in their accurate classification (A). Margin is the distance between the hyperplane and the support vectors. Therefore, the larger the margin, the better the data segregation and the lower the generalization error of the classifier. In large database sets with multiple features $(\mathbf{B}, \mathbf{C})$, data are mapped into a $3 \mathrm{D}$ configuration (kerneling method) and sheets are used as hyperplanes to classify them $(\mathbf{B}, \mathbf{C})$. $\mathrm{SVM}=$ support vector machine.
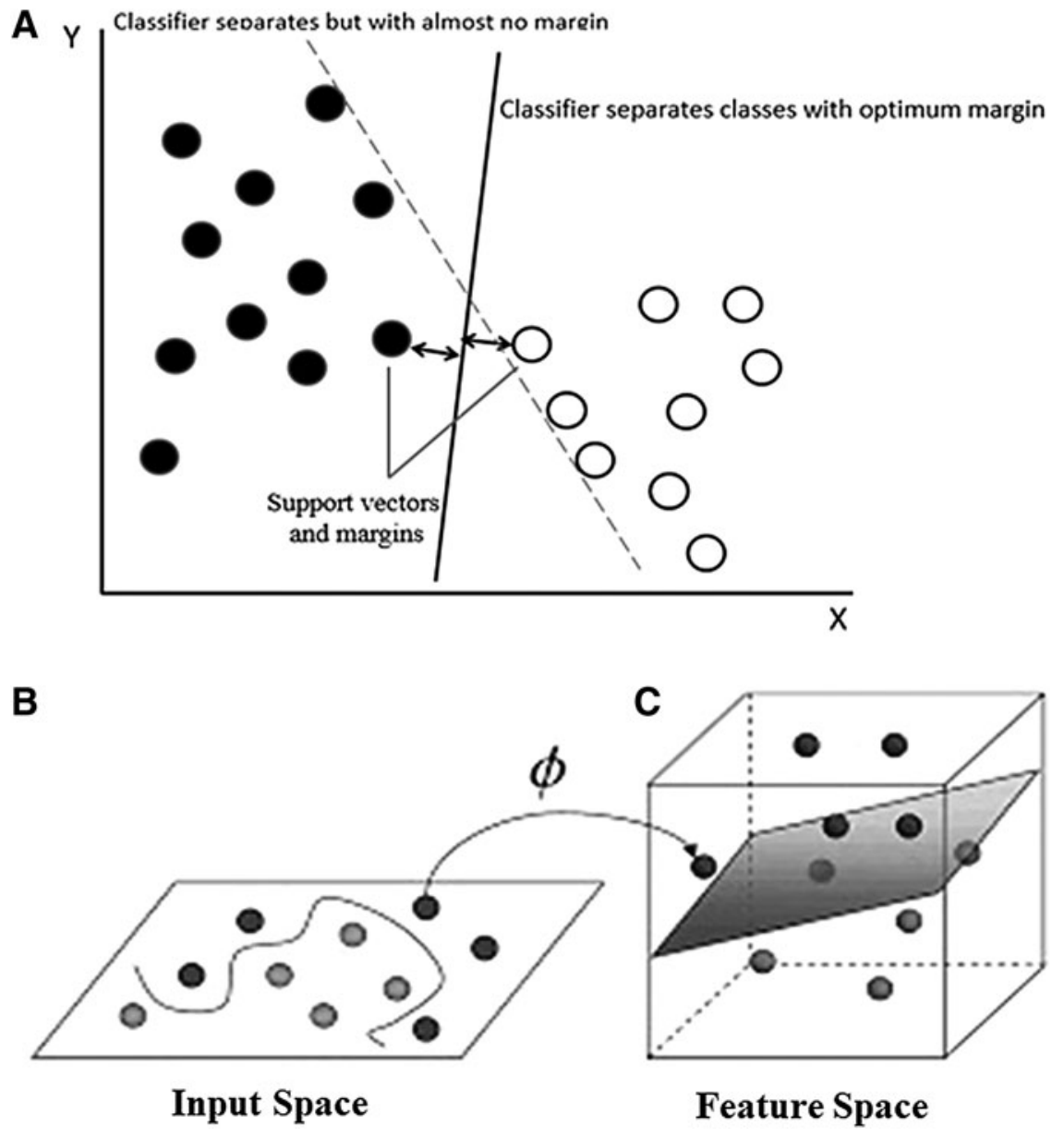
Table 1. Demographic, Preoperative, and Intraoperative Characteristics of Patients

Preoperative and intraoperative variables $(\mathrm{n}=146)$

Age (years), mean $\pm \mathrm{SD}$

Gender (male/female)

Stone burden $\left(\mathrm{mm}^{2}\right),{ }^{\mathrm{a}}$ mean $\pm \mathrm{SD}$

Stone size $(\mathrm{mm}),{ }^{\mathrm{b}}$ mean $\pm \mathrm{SD}$

Stone side (right/left)

Previous surgery in target kidney, $n(\%)$

History of diabetes, $n(\%)$

History of hypertension, $n(\%)$

Preoperative hemoglobin (g/dL), mean \pm SD

Preoperative creatinine $(\mathrm{mg} / \mathrm{dL})$, mean \pm SD

Body mass index $\left(\mathrm{kg} / \mathrm{m}^{2}\right)$, mean $\pm \mathrm{SD}$

Renal anomaly, $n(\%)$

Skeletal anomaly, $n(\%)$

Stone location, $n(\%)$

Upper calix

Mid calix

Lower calix

Renal pelvis

Staghorn

Multiple

$49.3 \pm 12.65$

$87 / 59$

$451.2 \pm 427.8$

$30.23 \pm 15.8$

$68 / 78$

$22(15)$

$19(13)$

$28(19.2)$

$13.84 \pm 1.9$

$1.2 \pm 0.6$

$26.8 \pm 5.13$

$7(4.8)$

$1(0.7)$

$6(4.1)$

2 (1.4)

$24(16.4)$

48 (32.9)

$26(17.8)$

40 (27.4)

Stone lucency, $n(\%)$

Opaque

Semiopaque

$73(50)$

$28(19.1)$

$45(30.8)$

Degree of hydronephrosis, $n(\%)$

Mild

$69(47.3)$

$61(41.8)$

16 (10.95)

Severe

$62(42.5)$

$46(31.5)$

$12(8.2)$

$26(17.8)$

Grade III

Grade IV

CROES nomogram score, $n(\%)$

100-129

130-169

170-219

$\geq 220$

$9(6.2)$

13 (8.9)

38 (26.0)

$86(58.9)$

Radiation exposure during access ${ }^{\mathrm{c}}$ (minutes), $\quad 1.43 \pm 1.69$ mean \pm SD

Operative time $^{\mathrm{d}}$ (minutes), mean $\pm \mathrm{SD}$

$37.5 \pm 20.7$

${ }^{\text {a }}$ Stone burden $=$ length $\times$ width $\times 0.78$.

${ }^{\mathrm{b}}$ Largest diameter.

${ }^{c}$ Defined as duration of X-ray exposure from insertion of the access needle to the start of nephroscopy.

${ }^{\mathrm{d}}$ From insertion of needle access to insertion of final nephrostomy.

CROES $=$ Clinical Research Office of Endourological Society.

software results with the actual outcomes to calculate the performance of the software for predicting each variable (Table 3). In general, the software predicted the PCNL outcomes with an accuracy ranging between $80 \%$ and $95.1 \%$. When post-PCNL stone-free status was evaluated according to GSS grades and CROES nomogram score, higher Guy's stone grades and lower CROES nomogram scores were significantly associated with a lower SFR ( $p=0.01$ and $p=0.03$, respectively). Figure 3 shows the stone-free status according to each classification system. When ROC curves were plotted for each predictive model for stone-free status (Fig. 4), the
Table 2. Postoperative Outcomes in the Cohort of Patients with Percutaneous Nephrolithotomy

Postoperative outcome variables $(\mathrm{n}=146)$

Stone free, $n(\%)$

Postoperative stone burden $\left(\mathrm{mm}^{2}\right),{ }^{a}$

$106(72.6)$

mean \pm SD

Postoperative hemoglobin $(\mathrm{g} / \mathrm{dL})$, mean \pm SD $11.6 \pm 1.26$

Postoperative creatinine (mg/dL), mean \pm SD $1.21 \pm 0.28$

Postoperative urine leakage (URS+Double-J 12 (8.2) stent insertion), $n(\%)$

Need for blood transfusion, $n(\%)$

Postoperative ancillary procedures ${ }^{\mathrm{b}}, n(\%)$

SWL

PCNL

$31(21.2)$

$11(7.5)$

${ }^{\text {a }}$ Stone burden $=$ length $\times$ width $\times 0.78$.

${ }^{\mathrm{b}}$ Two patients received both procedures (SWL+subsequent PCNL) for management of their residual stones.

$\mathrm{PCNL}=$ percutaneous nephrolithotomy; $\mathrm{SWL}=$ extracorporeal shockwave lithotripsy; URS = ureteroscopy.

AUC for the machine learning software (0.915) was significantly larger than the AUC for GSS (0.615) or CROES (0.621) nomograms $(p<0.001)$. The machine learning system recognized stone burden, the presence of staghorn or multiple renal stones as the most highly weighted preoperative factors affecting the post-PCNL SFR.

\section{Discussion}

In the era of minimally invasive stone surgery, PCNL has been considered the standard of care for managing large renal stones. According to a global study conducted by CROES, the overall stone-free and complication rates after PCNL were $75.5 \%$ and $20.5 \%$, respectively, with stone burden and morphometry as the main predictors of SFR. ${ }^{12}$ In recent years, several scoring systems have been developed for the prognostic evaluation of PCNL. These predictive models are important for patient counseling, patient selection (e.g., risk adjustment and referral to tertiary centers), and the evaluation of the quality of care and treatment efficacy. The GSS grades and CROES nomogram were originally reported in 2011 and 2013, respectively. 3,4

As a simple subjective and reproducible scoring system, GSS can be used to categorize patients into four grades based on their stone burden, configuration, and the presence of kidney or skeletal anomalies. ${ }^{3,13}$ Several case series validated the performance of this system to predict post-PCNL stonefree status $(\mathrm{AUC}=0.69-0.79) .{ }^{14-16}$ Yet despite its efficacy, GSS was originally developed by qualifying image analyses based on expert opinion, not on data-driven analyses. Obviously, there are a number of preoperative and intraoperative variables that cannot be processed by this system.

On the other hand, the CROES nomogram is a data-driven model based on a global cohort of patients from 96 centers worldwide. $^{4,12}$ This system calculates multiple regression analyses, several preoperative variables, and the relative weight of each input, and it uses them to predict post-PCNL SFR. $^{4,12,13}$ Since this nomogram is based on global data, understandably, several groups subsequently validated the CROES nomogram in light of its acceptable performance in predicting SFR (AUC $=0.641-0.76) .^{4,17-20}$ However, as the 
Table 3. Performance of the Machine Learning-Based Software in Predicting Stone-Free Status, Need for Blood Transfusion, and Different Ancillary Procedures in the Cohort

\begin{tabular}{lcccc}
\hline & $\begin{array}{c}\text { True positive rate } \\
\text { (sensitivity) }\end{array}$ & $\begin{array}{c}\text { False positive } \\
\text { rate }\end{array}$ & $\begin{array}{c}\text { Accuracy } \\
(\%)\end{array}$ & $\begin{array}{c}\text { Precision (positive predictive } \\
\text { value) }\end{array}$ \\
\hline Stone-free status & 0.92 & 0.11 & 91.8 & 0.95 \\
Need for repeat PCNL & 0.97 & 0.34 & 95.1 & 0.95 \\
Need for SWL & 0.82 & 0.48 & 80.0 & 0.81 \\
Need for URS and stent & 0.91 & 0.38 & 91.1 & 0.91 \\
insertion & & 0.3 & 83.0 & 0.82 \\
Need for blood transfusion & 0.89 & & & \\
\hline
\end{tabular}

${ }^{\mathrm{a}}$ To manage urine leakage.

developers acknowledged, ${ }^{4,13}$ several important preoperative variables are not considered in this model. Moreover, the CROES nomogram is admittedly complex and its application may not be practical at high-volume or nonacademic centers. $^{13,20}$

Several studies have compared the predictive performance of GSS as a quick qualitative measure versus the CROES nomogram as a complex quantitative model. ${ }^{20}$ In general, these studies showed a comparable performance of GSS grading $(\mathrm{AUC}=0.629-0.821)$ versus the CROES nomogram $(A U C=0.627-0.820)$ in predicting post-PCNL SFR ${ }^{21-27}$ In a systematic review, Withington and colleagues showed that although the validity of GSS is supported by a marginally higher quality of evidence compared with other nomograms, in general, the performance of all systems is similar for stonefree status. However, their questionable efficacy for predicting post-PCNL adverse events called attention to the need for further improvement. ${ }^{28}$
The use of machine learning approaches is advancing in urology, particularly in the fields of uro-oncology and urolithiasis. Appropriately trained machine learning systems can be exposed to new inputs, which can endow them with the capacity for continuous learning and improve their ability to recognize patterns and associations between variables. ${ }^{29}$ In 2017, we reported the accuracy of an artificial neural network (ANN) algorithm (ranging between $81.0 \%$ and $98.2 \%$, AUC $=0.861$ ) in predicting SFR, the need for post-PCNL ancillary procedures, and the need for blood transfusion. ${ }^{6}$ Supervised learning algorithms have previously been used to adjust weight vectors and classifiers. ${ }^{6}$ In this study, we set out to validate the accuracy of our adequately trained SVMbased software in processing prospective data from a new cohort. After about 2 years of the application of these intelligent systems in our practice, we consistently observed a high performance of them in predicting stone-free status in the current cohort $(\mathrm{AUC}=0.915)$ as well as in our initial report
FIG. 3. The stone-free rate in each subgroup of Guy's stone score grades and the CROES nomogram.

CROES = Clinical Research Office of Endourological Society.

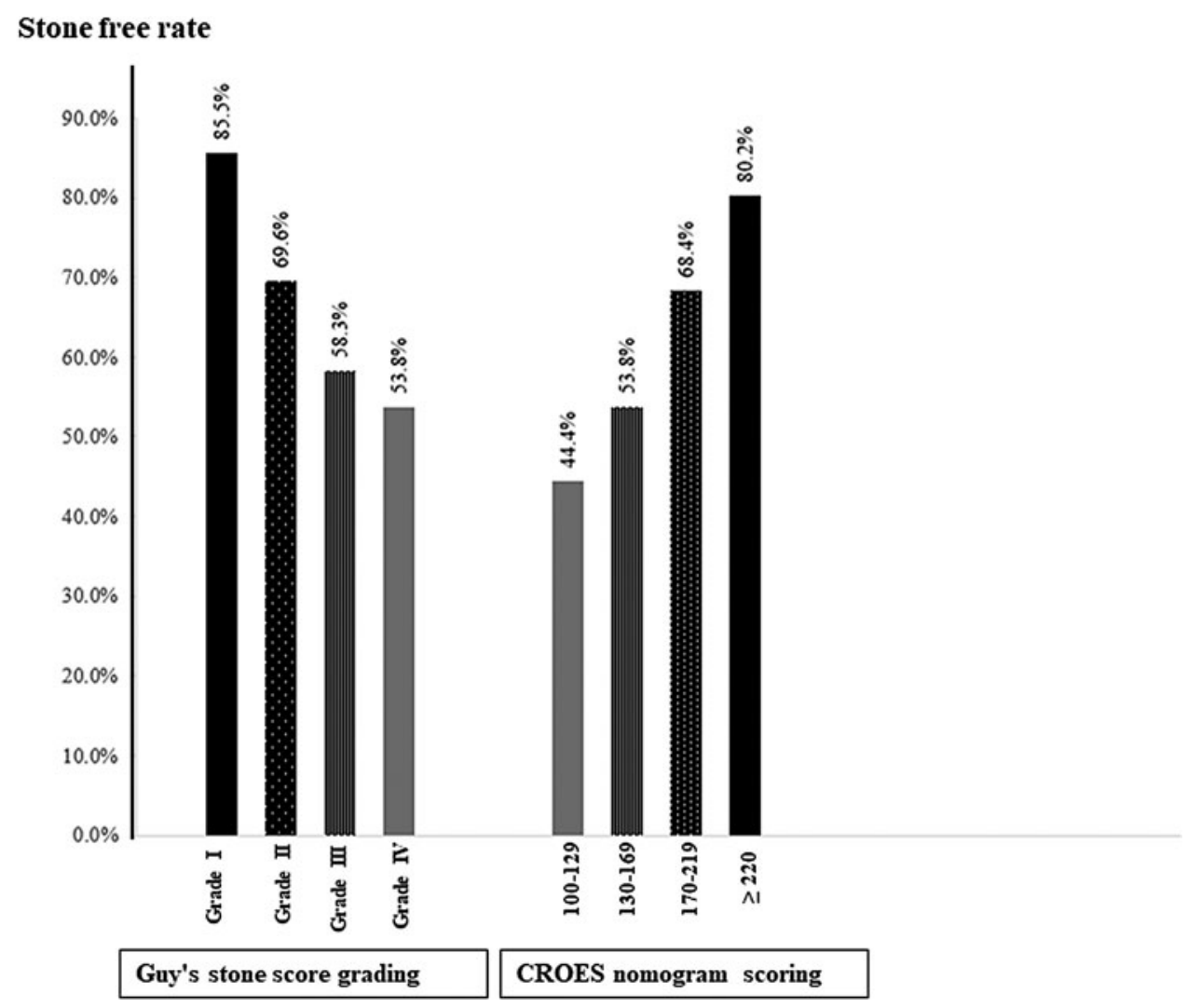




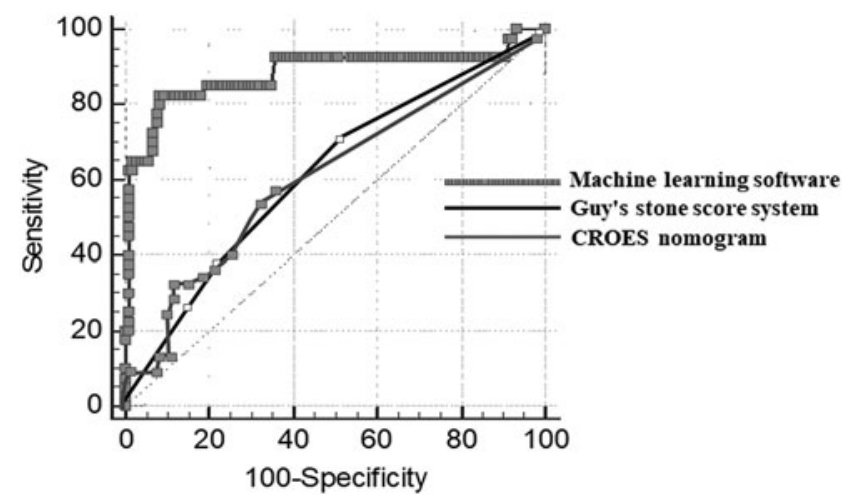

FIG. 4. ROC curve for stone-free status. The AUC for the machine learning software (0.915) was significantly larger than the AUC for Guy's gradings (0.615) and CROES (0.621) nomograms. AUC $=$ area under the ROC curve; $\mathrm{ROC}=$ receiver operating characteristic.

(AUC $=0.861) .{ }^{6}$ Not only was the predictive performance of the machine learning system better than the GSS grading and CROES systems; clearly, its intelligible digital interface facilitates application in almost any facility. Further, the machine learning software is also able to simultaneously process and report multiple endpoints. The system can also function as registry software to document a variety of current and predicted outputs. Previously, artificial intelligence systems were found to show similar or better efficacy than statistical data mining models in the evaluation of stone-free status after SWL. ${ }^{30,31}$ Nevertheless, additional comparative studies are needed to evaluate the prognostic accuracy of these modern predictive approaches compared with statistical models in the field of PCNL.

The ability to simultaneously predict the need for ancillary procedures and/or blood transfusion with the accuracy of $\geq 80 \%$ with intelligence systems is also acknowledgeable. Bleeding is the most significant complication of PCNL. All patients in this study had standard $26 \mathrm{~F}$ access to the collecting system. It has been shown that by reducing the tract size the risk of major bleeding may diminish with equal efficacy of stone clearance. ${ }^{32}$

Moreover, patients with miniperc- or microperc-PCNL may also be potential candidates for tubeless and ambulatory PCNL. ${ }^{32}$ With widespread implementation of the machine learning systems into these novel concepts of PCNL, patients' counseling regarding their postoperative morbidities and care pathways might be easier and more objective. This is also true if a digital intelligent system can predict the need for postoperative ancillary procedures to have an estimate about cost-effectiveness of the procedure beforehand. ${ }^{32,33}$ In the era of value-based health care, this information may be helpful not only for patients and caregivers but also for the administrators to balance the cost/surgical outcomes. The benefit of machine learning systems to improve efficiency of the health care system and optimizing the value of care remains a hot topic for further cost analysis studies.

This article presents, to our knowledge, the first comparative study of a machine learning digital application versus well-known nomograms currently used to predict PCNL outcomes. However, the limitations of our study need to be noted. When the machine learning systems process data algorithms, they may find and report associations that may not necessarily be meaningful or important for clinical practice. Therefore, frequent provider supervision and system evaluation are essential. We are also aware that this software is designed based on a "classical" PCNL procedure in adults. The conventional cutoff of $4 \mathrm{~mm}$ for significant postprocedural residual fragments was defined, which may not be optimal for calculation of "true" SFR ${ }^{33}$ and the outcome of patients with residual fragments $<4 \mathrm{~mm}$ was not captured.

For the next step, we are going to evaluate how this system can be used for "decision making" at our institute. Because of differences between institutes in procedural details, case volumes, and surgical experience, this preliminary step needs to be externally investigated and validated. Wider validation would be essential since we know that reporting from a single center may introduce bias.

Noteworthy advantages of machine learning-based software are that it can be easily updated, and more input and output variables and customized features can be added. Therefore, other clinical applications (e.g., patients amenable for ambulatory PCNL) may be addressed. Whether machine learning-based software is potentially applicable as a universal integrated decision-making tool that can be adopted by other centers and for other surgical procedures for urolithiasis remains a hot topic for future research.

\section{Acknowledgment}

The authors thank K. Shashok (AuthorAID in the Eastern Mediterranean) for improving the use of English in the article.

\section{Author Disclosure Statement}

No competing financial interests exist.

\section{Funding Information}

This article is based on the theses by S.T. and T.J.K. for the specialty degree in urology awarded by Shiraz University of Medical Sciences (grants no. 95-01-01-13978, 95-01-0113254).

\section{Supplementary Material}

Supplementary Table S1

\section{References}

1. Türk C, Petř́k A, Sarica K, Seitz C, Skolarikos A, Straub $\mathrm{M}$, Knoll T. EAU guidelines on interventional treatment for urolithiasis. Eur Urol 2016;69:475-482.

2. Zumstein V, Betschart P, Abt D, Schmid HP, Panje CM, Putora PM. Surgical management of urolithiasis-A systematic analysis of available guidelines. BMC Urol 2018; $18: 25$.

3. Thomas K, Smith NC, Hegarty N, Glass JM. The Guy's stone score-Grading the complexity of percutaneous nephrolithotomy procedures. Urology 2011;78:277-281.

4. Smith A, Averch TD, Shahrour K, Opondo D, Daels FP, Labate G, et al. CROES PCNL Study Group. A nephrolithometric nomogram to predict treatment success of percutaneous nephrolithotomy. J Urol 2013;190:149-156. 
5. Jeong CW, Jung JW, Cha WH, Lee BK, Lee S, Jeong SJ, et al. Seoul National University Renal Stone Complexity Score for predicting stone-free rate after percutaneous nephrolithotomy. PLoS One 2013;8:e65888.

6. Aminsharifi A, Irani D, Pooyesh S, Parvin H, Dehghani S, Yousofi K, Fazel E, Zibaie F. Artificial neural network system to predict the postoperative outcome of percutaneous nephrolithotomy. J Endourol 2017;31:461-467.

7. Aminsharifi A, Hosseini MM, Khakbaz A. Laparoscopic pyelolithotomy versus percutaneous nephrolithotomy for a solitary renal pelvis stone larger than $3 \mathrm{~cm}$ : A prospective cohort study. Urolithiasis 2013;41:493-497.

8. Harraz AM, Osman Y, El-Nahas AR, Elsawy AA, Fakhreldin I, Mahmoud O, El-Assmy A, Shokeir AA. Residual stones after percutaneous nephrolithotomy: Comparison of intraoperative assessment and postoperative non-contrast computerized tomography. World J Urol 2017;35:12411246.

9. Cortes C, Vapnik VN. Support-vector networks. Mach Learn 1995;20:273-297.

10. Ben-Hur A, Horn D, Siegelmann H, Vapnik VN. Support vector clustering, J Mach Learn Res 2001;2:125-137.

11. Shabaniyan T, Parsaei H, Aminsharifi A, Movahedi MM, Jahromi AT, Pouyesh S, Parvin H. An artificial intelligence-based clinical decision support system for large kidney stone treatment. Australas Phys Eng Sci Med 2019;42:771-779.

12. de la Rosette J, Assimos D, Desai M, Gutierrez J, Lingeman J, Scarpa R, Tefekli A. CROES PCNL Study Group. The Clinical Research Office of the Endourological Society Percutaneous Nephrolithotomy Global Study: Indications, complications, and outcomes in 5803 patients. J Endourol 2011;25:11-17.

13. Vernez SL, Okhunov Z, Motamedinia P, Bird V, Okeke Z, Smith A. Nephrolithometric scoring systems to predict outcomes of percutaneous nephrolithotomy. Rev Urol 2016;18:15-27.

14. de Souza Melo PA, Vicentini FC, Beraldi AA, Hisano M, Murta CB, de Almeida Claro JF. Outcomes of more than 1000 percutaneous nephrolithotomies and validation of Guy's stone score. BJU Int 2018;121:640-646.

15. Vicentini FC, Marchini GS, Mazzucchi E, Claro JF, Srougi M. Utility of the Guy's stone score based on computed tomographic scan findings for predicting percutaneous nephrolithotomy outcomes. Urology 2014;83:1248-1253.

16. Sfoungaristos S, Lorber A, Gofrit ON, Yutkin V, Landau EH, Pode D, Duvdevani M. External validation and predictive accuracy assessment of Guy's stone score as a preoperative tool for estimating percutaneous nephrolithotomy outcomes. J Endourol 2015;29:1131-1135.

17. Sfoungaristos S, Gofrit ON, Yutkin V, Landau EH, Pode D, Duvdevani M. External validation of CROES nephrolithometry as a preoperative predictive system for percutaneous nephrolithotomy outcomes. J Urol 2016;195: 372-376.

18. Sfoungaristos S, Mykoniatis I, Isid A, et al. Interobserver reliability and reproducibility of the Clinical Research Office of the Endourological Society nomogram in predicting percutaneous nephrolithotomy results. Urology 2016;97:56-60.

19. Sfoungaristos S, Gofrit ON, Pode D, Landau EH, Duvdevani M. Percutaneous nephrolithotomy for staghorn stones: Which nomogram can better predict postoperative outcomes? World J Urol 2016;34:1163-1168.
20. Vicentini FC, Serzedello FR, Thomas K, Marchini GS, Torricelli FCM, Srougi M, Mazzucchi E. What is the quickest scoring system to predict percutaneous nephrolithotomy outcomes? A comparative study among S.T.O.N.E score, Guys stone score and CROES nomogram. Int Braz J Urol 2017;43:1102-1109.

21. Labadie K, Okhunov Z, Akhavein A, et al. Evaluation and comparison of urolithiasis scoring systems used in percutaneous kidney stone surgery. J Urol 2015;193:154159.

22. Bozkurt IH, Aydogdu O, Yonguc T, Yarimoglu S, Sen V, Gunlusoy B, Degirmenci T. Comparison of Guy and Clinical Research Office of the endourological society nephrolithometry scoring systems for predicting stone-free status and complication rates after percutaneous nephrolithotomy: A single center study with 437 cases. J Endourol 2015;29:1006-1010.

23. Yarimoglu S, Bozkurt IH, Aydogdu O, Yonguc T, Sefik E, Topcu YK, Degirmenci T. External validation and comparison of the scoring systems (S.T.O.N.E, GUY, CROES, $\mathrm{S}-\mathrm{ReSC}$ ) for predicting percutaneous nephrolithotomy outcomes for staghorn stones: A single center experience with 160 cases. Kaohsiung J Med Sci 2017;33:516-522, 523.

24. Tailly TO, Okhunov Z, Nadeau BR, et al. Multicenter external validation and comparison of stone scoring systems in predicting outcomes after percutaneous nephrolithotomy. J Endourol 2016;30:594-601.

25. Choi SW, Bae WJ, Ha US, Hong SH, Lee JY, Kim SW, Cho HJ. Prediction of stone-free status and complication rates after tubeless percutaneous nephrolithotomy: A comparative and retrospective study using three stonescoring systems and preoperative parameters. World J Urol 2017;35:449-457.

26. Kumar S, Sreenivas J, Karthikeyan VS, Mallya A, Keshavamurthy R. Evaluation of CROES nephrolithometry nomogram as a preoperative predictive system for percutaneous nephrolithotomy outcomes. J Endourol 2016;30: 1079-1083.

27. Choi SW, Bae WJ, Ha US, Hong SH, Lee JY, Kim SW, Cho HJ. Prognostic impact of stone-scoring systems after percutaneous nephrolithotomy for staghorn calculi: A single center's experience over 10 years. J Endourol 2016;30: 975-981.

28. Withington J, Armitage J, Finch W, Wiseman O, Glass J, Burgess N. Assessment of stone complexity for PCNL: A systematic review of the literature, how best can we record stone complexity in PCNL? J Endourol 2016;30: 13-23.

29. Hinton G. Deep learning-A technology with the potential to transform health care. JAMA 2018;320:11011102.

30. Gomha MA, Sheir KZ, Showky S, Abdel-Khalek M, Mokhtar AA, Madbouly K. Can we improve the prediction of stone-free status after extracorporeal shock wave lithotripsy for ureteral stones? A neural network or a statistical model? J Urol 2004;172:175-179.

31. Seckiner I, Seckiner S, Sen H, Bayrak O, Dogan K, Erturhan S. A neural network-based algorithm for predicting stone-free status after ESWL therapy. Int Braz J Urol 2017; 43:1110-1114.

32. Ganpule AP, Vijayakumar M, Malpani A, Desai MR. Percutaneous nephrolithotomy (PCNL) a critical review. Int J Surg 2016;36(Pt D):660-664. 
33. Emmott AS, Brotherhood HL, Paterson RF, Lange D, Chew BH. Complications, re-intervention rates, and natural history of residual stone fragments after percutaneous nephrolithotomy. J Endourol 2018;32:28-32.

Address correspondence to:

Alireza Aminsharifi, $M D$

Department of Urology

Shiraz University of Medical Sciences

Zand Street

PO Box 71344

Shiraz, Iran

E-mail: aminsha@ccf.org; aminsharifi_ar@yahoo.com

$\quad$ Abbreviations Used
ANN $=$ artificial neural network
AUC $=$ area under ROC curve
CROES $=$ Clinical Research Office of Endourological
$\quad$ Society
CT $=$ computed tomography
GSS $=$ Guy's stone score
PCNL $=$ percutaneous nephrolithotomy
ROC $=$ receiver operating characteristic
SFR $=$ stone-free rate
SVM $=$ support vector machine
SWL $=$ extracorporeal shockwave lithotripsy
$\mathrm{URS}=$ ureteroscopy

\title{
Editorial Comment on: \\ Predicting the Postoperative Outcome of Percutaneous Nephrolithotomy with Machine Learning System: Software Validation and Comparative Analysis with Guy's Stone Score and the CROES Nomogram by Aminsharifi et al. (J Endourol 2020;34(6):692-699; DOI: 10.1089/end.2019.0475)
}

\author{
Ahmed R. EL-Nahas, MD
}

$\mathbf{P}$ ERCUTANEOUS NEPHROLITHOTOMY (PCNL) is the best treatment for large and complex renal stones according to all major guidelines. The goal of PCNL is achieving a stone-free status with minimal morbidity. Prediction of PCNL outcomes is essential for patients' counseling and planning surgical approach. Researches in this field started with identification of risk factors for stone-free rate and complications. ${ }^{1}$ The second step was development and validation of different scores and nomograms. ${ }^{2-4}$ The third and most recent step was using artificial neural network to develop a machine-based learning system. ${ }^{5}$ It implied feeding the system with the preoperative and outcome data of patients who underwent PCNL (learning phase). The system will not only detect factors affecting a given outcome, but it will also calculate the weight of each factor (relative relevance). Then preoperative data were used by the system to predict certain outcomes in another group of patients (testing phase).

Authors in this study internally validated their system in predicting stone-free rate and need for auxiliary procedures by comparing its results with a scoring system (Guy's scoring system) and a nomogram (CROES nomogram). It was found that machine-based learning system was statistically better than both. ${ }^{6}$ Moreover, the system detected that stone burden (length $\times$ width $\times 0.78$ ), staghorn, and multiple stones had the highest weight in predicting stone-free rate. Another advantage of this system over scores and nomograms is its ability to predict outcomes (such as need for blood transfusion and auxiliary procedures) other than stone-free rate. They also designed a user-friendly interface for data registry that can be used in the future for collection of more cases, adding more input and output data. The 